\title{
Asteroid population models
}

\section{Alessandro Morbidelli}

Observatoire de la Côte d'Azur, Nice, France email: Alessandro.MORBIDELLI@obs-nice.fr

Despite of the large number of detections of new asteroids, both in the main belt and in the near-Earth space, our observational knowledge of the asteroid populations is not quite complete. Our catalogs are affected by observational biases. Thus, a modeling work is required in order to infer the real distributions of the asteroid populations.

In collaboration with scientists of the Southwest Research Institute in Boulder, the Universities of Hawaii and Prague and the Nice observatory, I have been heavily involved in the development of models of the de-biased distributions of main belt objects and NEAs. In this short paper, I will briefly summarize the principles on which our models are based and their main results, with references to the key papers that discuss these models in detail. I will end on a model of the Yarkovsky-driven origin of NEAs from the main belt, which links the two models together and enlightens their mutual compatibility.

The NEA population is made of transient objects, with quite short individual dynamical lifetimes (a few million years only; Gladman et al. 1997). But the NEA population has remained roughly constant over the last $\sim 3$ Gy (Grieve and Shoemaker, 1994). This argues that the population is maintained in a sort of steady state by the continuous influx of new objects from the main belt. From numerical simulations, we determined the steady state orbital distributions of the NEAs coming from the main source resonances in the belt (Bottke et al. 2000). The problem was then to compute the relative contributions of these various sources to the overall NEA population. We did this by fitting our combined model distribution (i.e. the linear combination of the distributions related to each of the sources) to the observed distribution. This required an accurate evaluation of observational biases. For this reason, we limited our observational sample to the NEAs detected by the Spacewatch survey, whose bias had been previously quantified (Jedicke, 1996). Our model accounts for the existence of $\sim 1200$ NEAs with absolute magnitude $H<18$. In order to keep the NEA population in steady state, about 55 new asteroids with $H<18$ should become NEAs per My from the $\nu_{6}$ resonance, and 100 from the 3:1 resonance. (Bottke et al. 2002). Knowing the albedo distribution of the main belt asteroids close/in the NEA sources, we also derived an estimate of the albedo distribution of the NEAs. This allowed us to convert the magnitude distribution into a size distribution and compute the impact probability of NEAs with the Earth, as a function of impact energy. We estimated the existence about $\sim 1000$ NEAs larger than $1 \mathrm{~km}$ in diameter, with an exponent of the size distribution equal to -1.75 . The average impact rate for energies larger than 1,000 MT should be of 1 collision every 55,000 y (Morbidelli et al. 2002a). We are currently in the process of revising our model, with an improved calibration of the relative contributions of the sources, done using the much larger sample of NEAs detected by the LINEAR survey. The results, however, do not seem to change significantly.

For the main belt population, we used a completely different strategy (Morbidelli and Vokrouhlicky, 2003). First, we adopted the cumulative luminosity function of the main belt provided by the SDSS survey (Ivezic et al. 2001), and normalized the counts at the bright end using the known object catalog. This gave a total population of 1,300,000 
asteroids with $H<18$. It implied that the observed population was complete up to $H \sim 14$. For $H>14$ we generated synthetic objects in order to complete the observed population. Two problems needed to be solved, though. The first problem was to decide, as a function of magnitude, which fraction of the synthetic objects should belong to one of the various dynamical families, and which to the background population. We did this using the relative de-biasing technique for families and local background populations, developed in Morbidelli et al. (2003). The second problem was to decide the orbital distribution of the synthetic objects, separately for family and background members. The analysis of families showed that the orbital distribution of the observed members broadens with increasing magnitude up to $H \sim 14-15$, and then it is roughly invariant. Given that all our synthetic objects have $H>14$, we distributed them according to these invariant distributions. For the background objects, we divided the main belt in semimajor axis zones, and assumed that the observed $e, i$ distributions are representative of the real ones. Then we placed our synthetic background objects on orbits drawn from these distributions. In our resulting main belt model, about $60 \%$ of the asteroids with $H<18$ are members of the background population. Once magnitudes are converted into sizes, the exponent of the size distribution for objects of about $1 \mathrm{~km}$ in diameter (or $H \sim 18)$ is -1.3 .

It has been generally believed that the collisional activity in the main belt, which continuously breaks-up large asteroids, is the main mechanism that supplies bodies to the NEA source resonances. The size distribution of the NEAs, however, is only moderately steeper than that on the main belt. It should be much steeper if the NEAs were fresh collisional debris. This suggests that Yarkovsky thermal drag, rather than collisional injection, plays the dominant role in delivering material to the NEA source resonances (Morbidelli et al. 2002b). In Morbidelli and Vokrouhlicky (2003), starting from our Main Belt model, assuming current wisdom lifetimes for asteroid collisional disruption and reorientation, and using state-of-the-art estimates for the magnitude of the Yarkovsky and of the YORP effects, we showed that the populations of objects delivered per million year to the $\nu_{6}$ and 3:1 resonances are in agreement - for what concerns total number and size distribution- with those deduced from our NEA model. This shows that (i) our main belt model is compatible with our NEA model and (ii) the Yarkovsky is the major mechanism by which main belt asteroids become NEAs.

\section{References}

Bottke W. F., Jedicke R., Morbidelli A., Petit J. M. \& Gladman B. 2000, Science, 288, 2190

Bottke W. F., Morbidelli A., Jedicke R., Petit J. M., Levison H. F., Michel P. \& Metcalfe T.S. 2001, Icarus, 156, 399

Gladman B., Migliorini F., Morbidelli A., Zappalà V., Michel P., Cellino A., Froeschlé Ch., Levison H., Bailey M. \& Duncan M. 1997, Science, 277, 197

Grieve R. A. \& Shoemaker E. M. 1994, in: T. Gehrels \& M. S. Matthews (eds.), Hazards Due to Comets and Asteroids, University of Arizona Press, p. 417

Ivezić Z. et al. 2001, Astron. J., 122, 2749

Jedicke R. 1996, Astron. J., 111, 970

Morbidelli A., Jedicke R., Bottke W. F., Michel P. \& Tedesco E. F. 2002a, Icarus, 158, 329

Morbidelli A., Bottke W. F., Froeschlé C. \& Michel P. 2002b, in: Bottke et al. (eds.), Asteroids III, University of Arizona Press, p. 409

Morbidelli A., Nesvorný D., Bottke W. F., Michel P., Vokrouhlický D. \& Tanga P. 2003, Icarus, 162,328

Morbidelli A. \& Vokrouhlický D. 2003, Icarus, 163, 120 